\title{
Electrical characterization of Mg-doped GaN grown by metalorganic vapor phase epitaxy
}

\author{
J. W. Huang ${ }^{\text {a) }}$ and T. F. Kuech \\ Department of Chemical Engineering, University of Wisconsin, Madison, Wisconsin 53706 \\ Hongqiang Lu and Ishwara Bhat \\ Electrical, Computer and Systems Engineering Department, Rensselaer Polytechnic Institute, \\ Troy, New York 12180
}

(Received 4 December 1995; accepted for publication 15 February 1996)

\begin{abstract}
We have applied frequency-dependent capacitance measurements and admittance spectroscopy on $\mathrm{GaN}: \mathrm{Mg}$ to study the electronic states associated with $\mathrm{Mg}$ doping. Metalorganic vapor phase epitaxy GaN:Mg samples with two different $\mathrm{Mg}$ doping levels were grown and thermally annealed in nitrogen. Lateral dot-and-ring Schottky diodes using Au/Ti were fabricated. Frequency-dependent measurements on these diodes show that the capacitance is reduced at a higher frequency, most likely due to the inability of a deep center to maintain an equilibrium ionization state under a high-frequency modulation. Admittance spectroscopy, in which the conductance is monitored as a function of temperature, verifies the existence of one impurity-related acceptor level in the higher $\mathrm{Mg}$-doped sample with an activation energy of $136 \mathrm{meV}$. For the lower Mg-doped sample, two acceptor levels at 124 and $160 \mathrm{meV}$ were observed. We believe these levels are most probably associated with the $\mathrm{Mg}$ acceptor state itself, possessing energy levels which are very close to the results previously reported in the literature. (C) 1996 American Institute of Physics. [S0003-6951(96)01617-9]
\end{abstract}

The successful $p$-type doping in metalorganic vapor phase epitaxy (MOVPE) GaN using Mg has allowed the realization of blue light emitting $p-n$ junction diodes. Treatments, such as electron-beam irradiation ${ }^{1}$ or thermal annealing, ${ }^{2}$ are usually required to activate the $p$-type conduction. Electrical characteristics of these Mg-related acceptors are, however, not well understood. The $\mathrm{Mg}$ acceptor binding energy has been reported to be 250 and $\sim 160 \mathrm{meV}$ from the donor-acceptor pair transition and from temperature-dependent photoluminescence (PL) experiments, respectively. ${ }^{3}$ The temperature-dependent Hall measurements revealed a Mg-related activation energy of 157 $\mathrm{meV} .{ }^{4}$ In this letter, we report the electrical characterization of $\mathrm{GaN}: \mathrm{Mg}$, using frequency-dependent capacitance measurements and admittance spectroscopy, in order to study the electronic states associated with $\mathrm{Mg}$ doping. It is well known that deep traps greatly affect the free carrier profiles, as determined by capacitance-voltage $(C-V)$ measurements. ${ }^{5}$ Given the aforementioned energy level depths associated with $\mathrm{Mg}$, as well as the fact that $\mathrm{Mg}$-doping is responsible for most or all of the observed $p$-type conductivity, the small signal capacitance of $\mathrm{Mg}$-doped $\mathrm{GaN}$ is expected to be dependent on measurement frequency. We had also found that deep level transient spectroscopy (DLTS) could not be successfully used to characterize these Mg-related deep levels, principally due to the low diode capacitance level at low measurement temperatures. In contrast, admittance spectroscopy is better suited for the study of these relatively shallow and/or fast levels. ${ }^{6}$

$\mathrm{Mg}$-doped GaN samples were grown in a horizontal MOVPE reactor at 100 Torr using trimethyl gallium (TMGa), bis-cyclopentadienyl magnesium $\left(\mathrm{Cp}_{2} \mathrm{Mg}\right)$, and

\footnotetext{
a)Present address: Hewlett-Packard Company, Optoelectronics Division, San Jose, CA 95131.
}

ammonia $\left(\mathrm{NH}_{3}\right)$ on (0001) sapphire substrates. ${ }^{7}$ The $\mathrm{Mg}$ doped layer, $\sim 2 \mu \mathrm{m}$ in thickness, was grown at $1050^{\circ} \mathrm{C}$ at a growth rate of $\sim 2 \mu \mathrm{m} / \mathrm{h}$. Two samples with different $\mathrm{Cp}_{2} \mathrm{Mg}$ flow rates at 0.72 (sample A) and 0.6 (sample B) $\mu \mathrm{mol} / \mathrm{min}$ were grown separately. After growth, both samples were annealed at $800{ }^{\circ} \mathrm{C}$ in a 600 Torr $\mathrm{N}_{2}$ ambient for $30 \mathrm{~min}$. Lateral dot-and-ring Schottky diodes using Ti/Au were fabricated. The dot electrode has a diameter of $560 \mu \mathrm{m}$, surrounded by a ring electrode with $40 \mu \mathrm{m}$ gap. The area of the ring electrode was greater than 10 times that of the dot. $C-V$ and frequency-dependent capacitance measurements were performed at room temperature, in the dark, using a Hewlett-Packard 4284A LCR meter in series mode with an ac modulation level at $15 \mathrm{mV}$ and a frequency, $f$, ranging from $100 \mathrm{~Hz}$ to $1 \mathrm{MHz}$. In admittance spectroscopy, the conductance $(G)$ as well as the susceptance $(j 2 \pi f C=j \omega C)$ were monitored over the temperature range of $80-300 \mathrm{~K}$.

We have measured the sample capacitance at a zero dc bias voltage as a function of frequency, as shown in Fig. 1, and found that the capacitance value was strongly frequency dependent. The observed variation in capacitance could be due to either the high resistivity of the film or the wellknown dispersion effect which occurs when a deep level is unable to follow the high-frequency voltage modulation and contribute to the net space charge in the depletion region. ${ }^{8}$ The former effect is relatively unimportant since the capacitance values in Fig. 1 were obtained from the series mode measurement of the LCR meter, in which the capacitance is determined independently of any series resistance. The lowfrequency capacitance should be dependent on the sum of deep and shallow impurities, while the high-frequency capacitance is given by the free carrier density. ${ }^{9}$ We have performed $C-V$ measurements at high $(1 \mathrm{MHz})$ and low (10 $\mathrm{kHz}$ ) frequencies to determine the net ionized impurity concentrations as shown in Fig. 2 for both samples. 


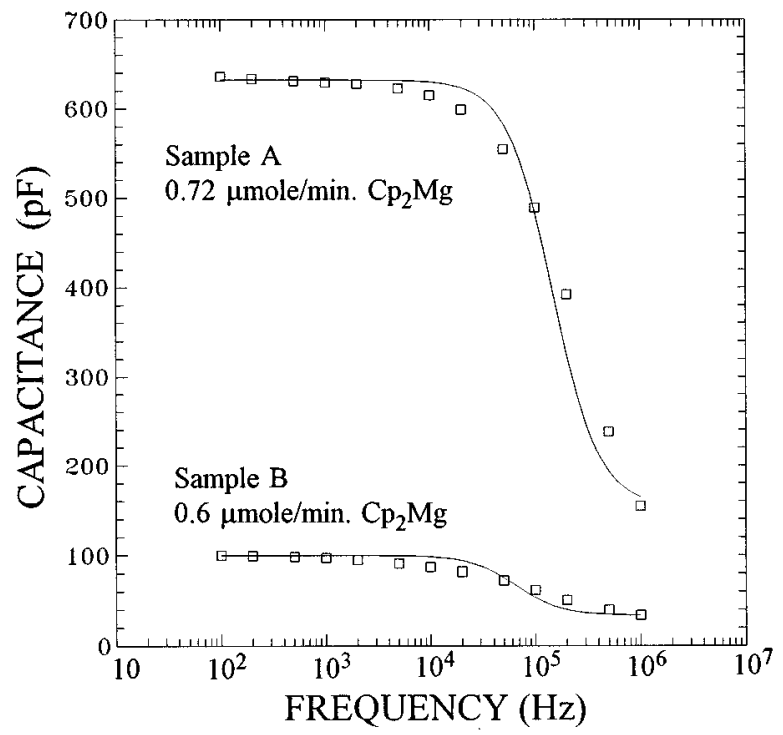

FIG. 1. Measured ( $\square$ ) and predicted (-) zero dc bias capacitance as a function of measurement frequency for samples A and B.

These two samples were also analyzed using admittance spectroscopy ${ }^{10,11}$ in order to investigate the deep levels associated with $\mathrm{Mg}$ doping. The admittance as a function of the measurement frequency, $\omega$, and temperature, $T$, is the sum of $G$ and $j \omega C$, where ${ }^{10}$

$$
G(\omega, T)=\frac{\omega_{t}\left(C_{0}-C_{\infty}\right)}{1+\left(\omega_{t} / \omega\right)^{2}}
$$

and

$$
C(\omega, T)=C_{\infty}+\frac{C_{0}-C_{\infty}}{1+\left(\omega / \omega_{t}\right)^{2}} .
$$

In Eqs. (1) and (2), $C_{0}$ and $C_{\infty}$ are low- and high-frequency capacitance values, respectively. The characteristic frequency, $\omega_{t}$, is proportional to the emission rate $\left(e_{p}\right)$ of free carriers from the deep level to a band edge state. At a fixed $\omega$, a peak in $G(\omega, T)$ as a function of $T$ is found to occur at $\omega_{t}=\omega$, where $C(\omega, T)$ is at the midpoint of a step change. ${ }^{11}$ Since

$$
\omega_{t}=\omega=2 \pi f \propto e_{p}=c_{p} N_{V} \exp (-\Delta E / k T),
$$

where $c_{p}$ is the hole capture coefficient of the deep level, $N_{V}$ is the valence band density of states, and $\Delta E$ is the depth of the deep level from the valence band edge. $\Delta E$ can be determined from the slope of $\log \left(T^{2} / f\right)$ vs $1 / T$ derived from measurements at several different $\omega$ to acquire the corresponding temperatures where $G$ is maximized at a given $\omega$. The admittance spectra of both GaN:Mg samples are shown in Fig. 3 at $10 \mathrm{kHz}$, with insets showing the determination of $\Delta E$. For sample $\mathrm{A}$, only one deep state was observed with $\Delta E$ at $136 \mathrm{meV}$, and the characteristic frequency associated with $\mathrm{Mg}$ acceptor at room temperature $(294 \mathrm{~K})$ was determined to be $\sim 148.4 \mathrm{kHz}$ from the $\log \left(T^{2} / f\right)$ vs $1 / T$ relationship. By using Eq. (2), the frequency dependence of capacitance at $294 \mathrm{~K}$ can be predicted and found to be in reasonably good agreement with the measured data as shown in Fig. 1. The $136 \mathrm{meV}$ energy level is very close to the reported $\mathrm{Mg}$ acceptor binding energy from Hall measure-
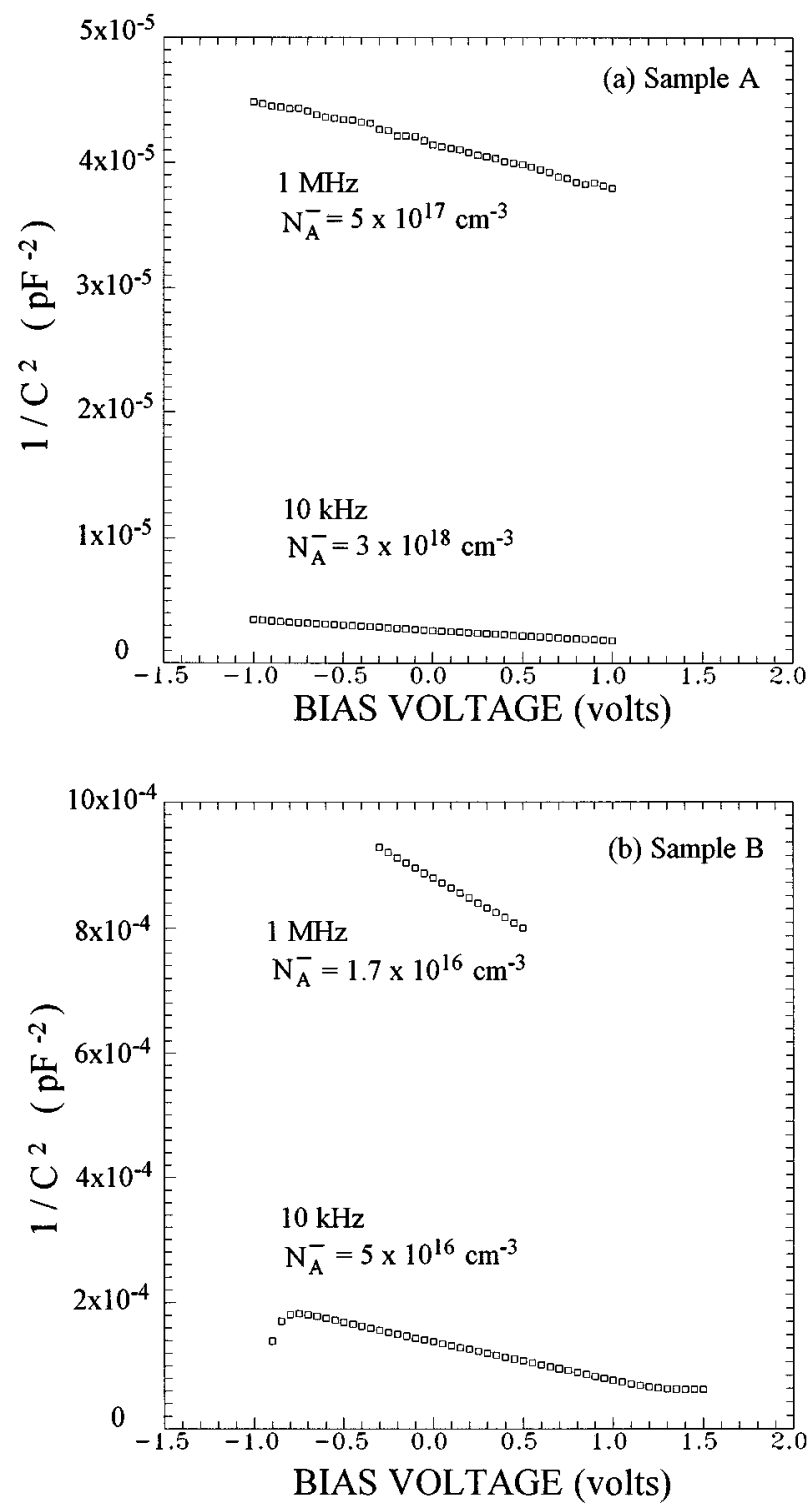

FIG. 2. The room-temperature capacitance-voltage characteristics of $\mathrm{Ti} / \mathrm{Au} /$ GaN:Mg Schottky contact for (a) sample A, and (b) sample B.

ment or PL, ${ }^{3,4}$ and would contribute to the low net ionized impurity concentration at $5 \times 10^{17} \mathrm{~cm}^{-3}$ as determined by $C-V$ at $1 \mathrm{MHz}$ [Fig. 2(a)]. In this high-frequency case, not all $\mathrm{Mg}$ acceptors can follow the fast voltage modulation used in the $C-V$ measurement and these $\mathrm{Mg}$ acceptors do not contribute to the measured change in the net space charge. The total $\mathrm{Mg}$ concentration can be calculated from the charge neutrality equation given by

$$
N_{A}^{-}=\frac{N_{A}}{1+g p / N_{V} \exp (\Delta E / k T)}=p .
$$

A total $\mathrm{Mg}$ concentration of $N_{A}=6.67 \times 10^{18} \mathrm{~cm}^{-3}$ was determined by assuming $p=5 \times 10^{17} \mathrm{~cm}^{-3}$ as measured in Fig. 2(a), $N_{V}=1.74 \times 10^{19} \mathrm{~cm}^{-3}, 4 E=136 \mathrm{meV}, T=294$ $\mathrm{K}$, degeneracy factor $g=2,{ }^{4}$ and neglecting the existence of any other additional shallow impurities. This $N_{A}$ is about a factor of 2 greater than the $3 \times 10^{18} \mathrm{~cm}^{-3}$ as determined by $C-V$ at $10 \mathrm{kHz}$ [Fig. 2(a)].

At the lower $\mathrm{Mg}$ doping concentration, two deep levels appeared in sample B at 160 and $124 \mathrm{meV}$. The characteris- 

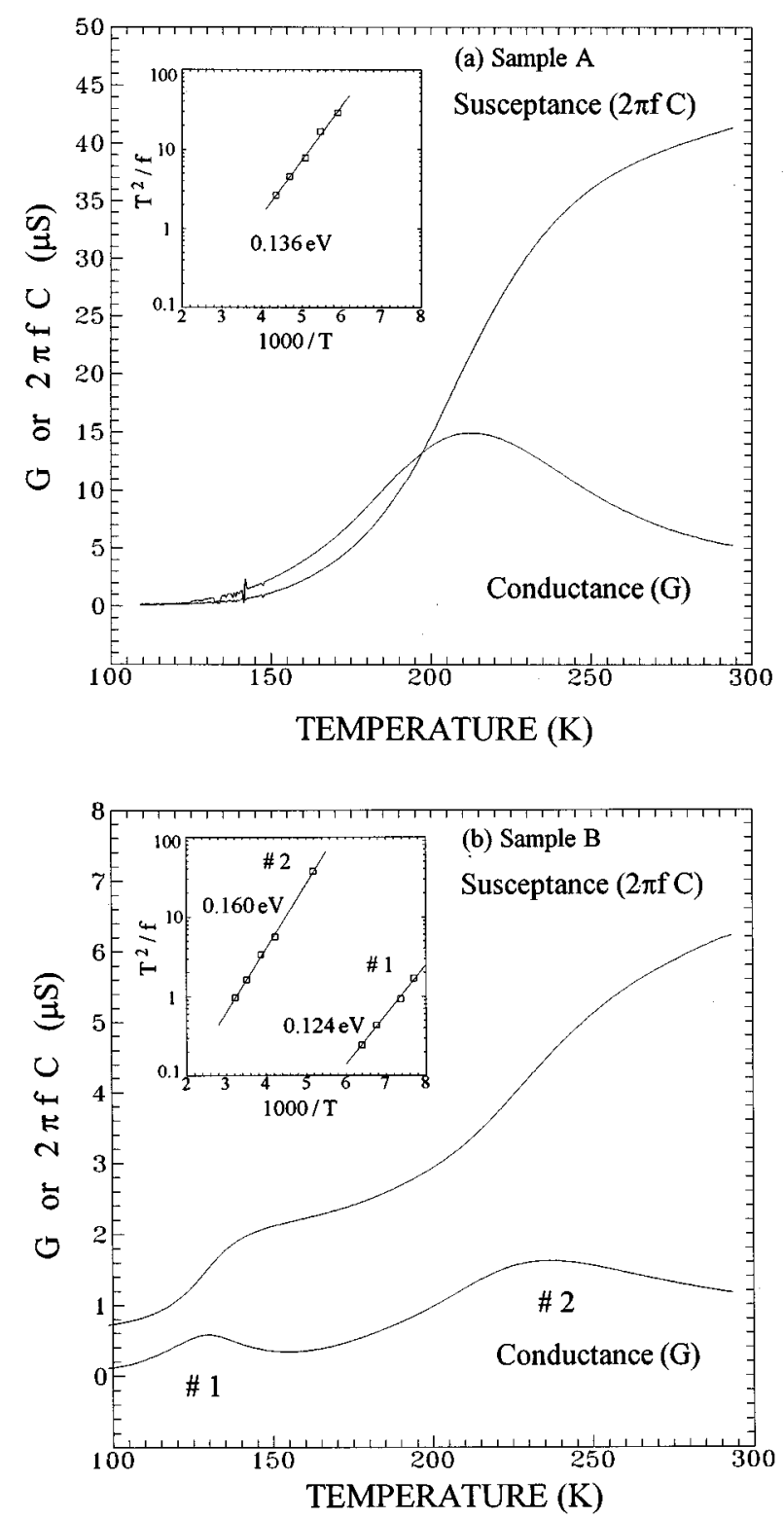

FIG. 3. Measured conductance and susceptance as a function of temperature for (a) sample A, and (b) sample B at $10 \mathrm{kHz}, 15 \mathrm{mV}$ modulation, and a zero dc bias voltage. The inset growth shows the determination of the activation energy.

tic frequency associated with the $160 \mathrm{meV}$ level at $294 \mathrm{~K}$ is $\sim 64.7 \mathrm{kHz}$ from the $\log \left(T^{2} / f\right)$ vs $1 / T$ relationship [Fig. $3(\mathrm{~b})]$. The agreement between the predicted and the measured $294 \mathrm{~K}$ frequency dependence of the capacitance, as shown in Fig. 1, is quite good through the use of this single characteristic frequency in Eq. (2). An extension of Eq. (2) to include both deep levels should lead to an even better agreement. ${ }^{10} N_{A}$, calculated through Eq. (4) using $\Delta E=160 \mathrm{meV}$ and $p=1.7 \times 10^{16} \mathrm{~cm}^{-3}$ from Fig. 2(b) at a frequency of $1 \mathrm{MHz}$, is $3.5 \times 10^{16} \mathrm{~cm}^{-3}$, which compares favorably with the measured $10 \mathrm{kHz}$ value of 5 $\times 10^{16} \mathrm{~cm}^{-3}$. The calculated values of $N_{A}$ in samples A and $\mathrm{B}$ are in relatively good agreement with experimental values in light of the simple model used to derive these quantities.

The 136 and $160 \mathrm{meV}$ energy levels of samples A and B, with a high and low $\mathrm{Mg}$ doping level, respectively, derived from our admittance spectroscopy measurements appear to be in close agreement to the results of a Hall measurement study by Tanaka $e t ~ a l .{ }^{4}$ They reported the activation energies of GaN:Mg to be 125 and $157 \mathrm{meV}$ for two samples from the same growth run but annealed for 60 and $25 \mathrm{~s}$, respectively. The $60 \mathrm{~s}$ annealed sample had more electrically active acceptors, similar to the case of our sample A. We have performed secondary ion mass spectroscopy (SIMS) analysis on our two GaN:Mg samples. Due to the lack of a GaN:Mg standard, only relative counts were available for the concentration of Mg. Sample A has about a factor of 6 greater $\mathrm{Mg}$ concentration than sample B. This observation is quite different from the ratio of electrically active species in these two samples of $3 \times 10^{18} / 5 \times 10^{16}=60$ as determined by $C-V$ measurements at low frequency (Fig. 2 with $f=10$ $\mathrm{kHz}$ ). This result suggests that there are more electrically active $\mathrm{Mg}$ acceptors in sample $\mathrm{A}$ than in B. It has been proposed that hydrogen passivation is related to the hole compensation mechanism. ${ }^{12}$ In previous studies, the amount of hydrogen was found to increase linearly with the Mg concentration in the as-grown MOVPE GaN:Mg samples. ${ }^{13}$ After annealing, the hydrogen density would decrease in general, but the exact relationship between the amount of hydrogen extraction, the $\mathrm{Mg}$ doping level, and the annealing time is not presently clear. ${ }^{14} \mathrm{~A}$ recent Raman and infrared absorption spectroscopic study by Brandt et al. ${ }^{15}$ has demonstrated the existence of four local vibrational modes for samples of GaN:Mg possessing a high concentration of hydrogen. One pair of the modes, with room-temperature frequencies of 2168 and $2219 \mathrm{~cm}^{-1}$, is assigned to $\mathrm{Mg}-\mathrm{H}$ complexes in the $c$ plane and parallel to the $c$ axis. A reduced hydrogen content may play a role in the presence of the two Mg-related deep levels, as well as the lower level of electrically active $\mathrm{Mg}$ acceptor in our sample B.

The authors would like to thank K. L. Bray for several useful discussions. The financial support of this work at the University of Wisconsin was provided by the Army Research Office and the National Science Foundation. The work at Rensselaer Polytechnic Institute was partially supported by Philips Laboratories, Briarcliff Manor, NY, and by Texas Instruments.

${ }^{1}$ H. Amano, M. Kito, K. Hiramatsu, and I. Akasaki, Jpn. J. Appl. Phys. 28, L2112 (1989).

${ }^{2}$ S. Nakamura, T. Mukai, M. Senoh, and N. Iwasa, Jpn. J. Appl. Phys. 31, L139 (1992)

${ }^{3}$ S. Fischer, C. Wetzel, E. E. Haller, and B. K. Meyer, Appl. Phys. Lett. 67, 1298 (1995)

${ }^{4}$ T. Tanaka, A. Watanabe, H. Amano, Y. Kobayashi, I. Akasaki, S. Yamazaki, and M. Koike, Appl. Phys. Lett. 65, 593 (1994).

${ }^{5}$ L. C. Kimerling, J. Appl. Phys. 45, 1839 (1974).

${ }^{6}$ D. L. Losee, J. Appl. Phys. 46, 2204 (1975).

${ }^{7}$ H. Lu and I. Bhat, Mater. Res. Soc. Symp. Proc. Vol. 395 (to be published).

${ }^{8}$ G. H. Glover, Solid-State Electron. 16, 973 (1973).

${ }^{9}$ E. Schibli and A. G. Milnes, Solid-State Electron. 11, 323 (1968).

${ }^{10}$ W. G. Oldham and S. S. Naik, Solid-State Electron. 15, 1085 (1972).

${ }^{11}$ H. C. Casey, A. Y. Cho, D. V. Lang, E. H. Nicollian, and P. W. Foy, J. Appl. Phys. 50, 3484 (1979).

${ }^{12}$ S. Nakamura, N. Iwasa, M. Senoh, and T. Mukai, Jpn. J. Appl. Phys. 31, 1258 (1992).

${ }^{13}$ Y. Ohba and A. Hatano, Jpn. J. Appl. Phys. 33, L1367 (1994).

${ }^{14}$ W. Götz, N. M. Johnson, J. Walker, D. P. Bour, and R. A. Street, Appl. Phys. Lett. 68, 667 (1996).

${ }^{15}$ M. S. Brandt, J. W. Ager, W. Götz, N. M. Johnson, J. S. Harris, R. J. Molnar, and T. D. Moustakas, Phys. Rev. B 49, 14758 (1994). 